\title{
A note on minimum linear arrangement for BC graphs
}

\author{
Xiaofang Jiang ${ }^{1}$, Qinghui Liu ${ }^{1, *}$, N. Parthiban ${ }^{2, \dagger}$ and R. Sundara Rajan ${ }^{3}$ \\ ${ }^{1}$ Department of Computer Science, Beijing Institute of Technology, \\ Beijing, China \\ ${ }^{2}$ School of Advanced Sciences, VIT University, Chennai, India \\ ${ }^{3}$ Department of Mathematics, Hindustan Institute of Technology and Science, \\ Chennai, India \\ parthiban24589@gmail.com
}

\begin{abstract}
A linear arrangement is a labeling or a numbering or a linear ordering of the vertices of a graph. In this paper we solve the minimum linear arrangement problem for bijective connection graphs (for short BC graphs) which include hypercubes, Möbius cubes, crossed cubes, twisted cubes, locally twisted cube, spined cube, $Z$-cubes, etc. as the subfamilies.
\end{abstract}

Keywords: Minimum linear arrangement, BC graphs

\section{Introduction}

Graph layout problems are a particular class of combinatorial optimization problems whose goal is to find a linear layout of an input graph in such a way that a certain objective function is optimized. In the literature, there are plenty of layout problems are discussed, such as Linear Arrangement, Bandwidth, Cutwidth, Modified Cut, Sum Cut, Edge Bisection and Vertex Bisection [1. A large number of relevant problems in different domains formulated as graph layout problems include VLSI circuit design, network reliability, information retrieval, numerical analysis, computational biology, single machine job scheduling, automatic graph drawing and topology awareness of overlay networks [2, 3]. The problems are hard in general but known to be solvable in certain restricted classes of graphs [1].

A linear arrangement $f$ of an undirected graph $G=(V, E)$ with $n$ nodes is a bijective function $f: V \rightarrow\{1,2, \ldots, n\}$. A linear arrangement is also called a labeling or a numbering or a linear ordering of the vertices of a graph. In [4, the Minimum Linear Arrangement (MinLA) problem is formulated as follows: Given a graph $G=(V, E)$, find a linear arrangement $f$ that minimizes $\sum_{(u, v) \in E}|f(u)-f(v)|$. Linear arrangements are a particular case of embedding graphs in $d$ dimensional grids or other graphs. The case in which a graph with $n$ vertices must be embedded into a path $P_{n}$ is perhaps the simplest nontrivial embedding problem. The MinLA problem is NP-complete for bipartite graphs [5] and permutation graphs [6].

\footnotetext{
${ }^{*}$ This work is partially supported by National Natural Science Foundation of China, No. 11371055, No. 11571030.

$\dagger$ This work is partially supported by Project no. SR/S4/MS: 846/13, Department of Science and Technology, SERB, Government of India
} 


\section{Preliminaries}

The following edge isoperimetric problems are used as tools to solve the MinLA problem. MinLA has been computed for regular graphs such as hypercubes [4], circulant graphs [8], folded hypercubes [7], Petersen graphs [9] chord graphs [3] and locally twisted cubes [10] using edge isoperimetric problem. In this paper, we compute the MinLA for certain families of regular graphs such as BC graphs.

Problem $1:$ [1] For a given $m$, if $\theta_{G}(m)=\min _{A \subseteq V,|A|=m}\left|\theta_{G}(A)\right|$ where $\theta_{G}(A)=\{(u, v) \in E: u \in$ $A, v \notin A\}$, then the problem is to find $A \subseteq V$ with $|A|=m$ such that $\theta_{G}(m)=\left|\theta_{G}(A)\right|$.

Problem $2:$ [11] For a given $m$, if $I_{G}(m)=\max _{A \subset V,|A|=m}\left|I_{G}(A)\right|$ where $I_{G}(A)=\{(u, v) \in E$ : $u, v \in A\}$, then the problem is to find $A \subseteq V$ with $|A|=m$ such that $I_{G}(m)=\left|I_{G}(A)\right|$. Such a set $\mathrm{A}$ is called an optimal set.

Definition 2.1. Let $G$ and $H$ be finite graphs. An embedding of $G$ into $H$ is a pair $\left(f, P_{f}\right)$ defined as follows:

1. $f$ is a one-to-one map from $V(G)$ to $V(H)$

2. $P_{f}$ is a one-to-one map from $E(G)$ to $\left\{P_{f}(u, v): P_{f}(u, v)\right.$ is a path in $H$ between $f(u)$ and $f(v)$, for $(u, v) \in E(G)\}$.

For brevity, we denote the pair $\left(f, P_{f}\right)$ as $f$. The expansion of an embedding $f$ is the ratio of the number of vertices of $H$ to the number of vertices of $G$. In this paper, we consider embeddings with expansion one.

The congestion of an embedding $f$ of $G$ into $H$ is the maximum number of edges of the graph $G$ that are embedded on any single edge $e$ of $H$. Let $E C_{f}(e)$ denote the number of edges $(u, v)$ of $G$ such that $e$ is in the path $P_{f}(u, v)$ between $f(u)$ and $f(v)$ in $H$. In other words,

$$
E C_{f}(e)=\left|\left\{(u, v) \in E(G): e \in P_{f}(u, v)\right\}\right|
$$

where $P_{f}(u, v)$ denotes the path between $f(u)$ and $f(v)$ in $H$ with respect to $f$. Further, if $S$ is any subset of $E(H)$, then we define $E C_{f}(S)=\sum_{e \in S} E C_{f}(e)$.

Definition 2.2. The wirelength of an embedding $f$ of $G$ into $H$ is given by

$$
W L_{f}(G, H)=\sum_{e \in E(H)} E C_{f}(e)
$$

The wirelength of $G$ into $H$ is defined as

$$
W L(G, H)=\min W L_{f}(G, H)
$$

where the minimum is taken over all embeddings $f$ of $G$ into $H$.

When $H$ is a path, we represent $W L_{f}(G, H)$ by $L A_{f}(G)$ and represent $W L(G, H)$ by $\operatorname{Min} L A(G)$.

Lemma 2.3. The MinLA of a graph $G$ of order $n$ is given by

$$
\operatorname{Min} L A(G) \geq \sum_{i=1}^{n-1} \theta_{G}(i) \text {. }
$$


Proof. For $1 \leq i<n$, let $S_{i}=(i, i+1)$, then for any embedding $f$, we have

$$
\begin{aligned}
E C_{f}\left(S_{i}\right) & \geq \theta_{G}(i) \\
\therefore \min _{f} \sum_{i=1}^{n-1} E C_{f}\left(S_{i}\right) & \geq \sum_{i=1}^{n-1} \theta_{G}(i) \\
\text { i.e., } \operatorname{MinL} A(G) & \geq \sum_{i=1}^{n-1} \theta_{G}(i) .
\end{aligned}
$$

\section{Main Results}

BC networks have received a great deal of attention in the past [12, 13, 14, 15. Fan et al. [12] proposed a family of interconnection networks called BC graphs. BC networks are a class of networks which include several well-known interconnection networks like hypercubes, Möbius cubes [16], crossed cubes [17], twisted cubes [18], locally twisted cube [19], spined cube [20] and Z-cube [21]. These variations of hypercubes generally possess certain superior properties over the hypercubes and are recognized as attractive alternatives to the hypercubes.

Definition 3.1. [12, 15] Let $G_{1}=\left(V_{1}, E_{1}\right), G_{2}=\left(V_{2}, E_{2}\right)$ be two vertex disjoint graph of the same order. A bijective connection between $G_{1}$ and $G_{2}$ is defined as an edge set $E=\{(v, \phi(v))\}$, where $\phi: V_{1} \rightarrow V_{2}$ is a bijection. Define $G_{1} \oplus G_{2}=\left(V_{1} \cup V_{2}, E_{1} \cup E_{2} \cup E\right)$.

An $n$-dimensional BC graph, denoted by $X_{n}$, is an $n$-regular graph with $2^{n}$ nodes and $n 2^{n-1}$ edges. The set of all the $n$-dimensional $\mathrm{BC}$ graphs is called the family of the $n$-dimensional $\mathrm{BC}$ graphs, denoted by $\ell_{n}$. We now define $X_{n}$ mathematically as follows:

Definition 3.2. [12, 15] The one-dimensional BC network $X_{1}$ is a complete graph with two vertices, $K_{2}$. The family of the one-dimensional BC network is defined as $\ell_{1}=\left\{K_{2}\right\}$. When $n \geq 2$, $G=X_{n} \in \ell_{n}$ if and only if $G=G_{1} \oplus G_{2}$ for some $G_{1}, G_{2} \in \ell_{n-1}$.

Lemma 3.3. [15] Let $G$ be a $n$-dimensional BC graph. For an integer $m$, which can be uniquely written as $m=\sum_{i=1}^{r-1} 2^{l_{i}}$ for some nonnegative integers $r$ and $l_{0}>l_{1}>\ldots>l_{r-1}$, then the maximum number of edges joining vertices from a set of $m$ vertices is $I_{G}(m)=\sum_{i=0}^{r-1}\left(l_{i} / 2+i\right) 2^{l_{i}}$, where $1 \leq$ $m \leq 2^{n}, n \geq 1$

Note that this implies $\theta_{G}(m)=n m-2 I_{G}(m)=\sum_{i=0}^{r-1}\left(n-l_{i}-2 i\right) 2^{l_{i}}$. And hence

$$
\sum_{m=1}^{2^{n}-1} \theta_{G}(m)=\sum_{k=0}^{n-1} 2^{k} \sum_{i=0}^{n-k-1}\left((n-k-2 i) 2^{k}\left(\begin{array}{c}
n-k-1 \\
i
\end{array}\right)\right)
$$

where for a sequence $n>l_{0}>l_{1}>\ldots>l_{r-1} \geq 0$ with $l_{i}=k$ for some $0 \leq i<n$, there are $2^{k}$ choices for $l_{i+1}, \ldots, l_{r-1}$ and $\left(\begin{array}{c}n-k-1 \\ i\end{array}\right)$ choices for $l_{0}, \ldots, l_{i-1}$. Then 


$$
\begin{aligned}
\sum_{m=1}^{2^{n}-1} \theta_{G}(m) & =\sum_{k=0}^{n-1} 2^{k}\left(2^{n-1}(n-k)-2^{k+1}(n-k-1) \sum_{i=1}^{n-k-1}\left(\begin{array}{c}
n-k-2 \\
i-1
\end{array}\right)\right) \\
& =\sum_{k=0}^{n-1} 2^{k}\left(2^{n-1}(n-k)-2^{k+1}(n-k-1) 2^{n-k-2}\right) \\
& =\sum_{k=0}^{n-1} 2^{n-1+k}=2^{n-1}\left(2^{n}-1\right) .
\end{aligned}
$$

Now, we define a class of linear arrangement from BC graph to path by induction.

Definition 3.4. For any $n \geq 0$, define $P_{2^{n}}=\left\{1,2, \cdots, 2^{n}\right\} \subset \mathbb{Z}$. Let $G=X_{n}$ be the $n$-dimensional $B C$ graph $G$. If $n=1$, writing $V(G)=\left\{v_{1}, v_{2}\right\}$, we define $f\left(v_{1}\right)=1, f\left(v_{2}\right)=2$. We call it a 1-dimensional $B C$ structure linear arrangement. If $n>1$, then there exist $(n-1)$-dimensional $B C$ graphs $G_{1}, G_{2}$ such that $G=G_{1} \oplus G_{2}$. Let $f_{1}: V\left(G_{1}\right) \rightarrow P_{2^{n-1}}, f_{2}: V\left(G_{2}\right) \rightarrow P_{2^{n-1}}$ be two $(n-1)$-dimensional BC structure linear arrangements. Define $f: V(G) \rightarrow P_{2^{n}}$ as follows. For any $v \in V\left(G_{1}\right)$, let $f(v)=f_{1}(v)$ and for any $v \in V\left(G_{2}\right)$, let $f(v)=f_{2}(v)+2^{n-1}$. We call it a $n$-dimensional $B C$ structure linear arrangement.

Theorem 3.5. The MinLA of $B C$ graph $X_{n}$ is

$$
\operatorname{MinL} A\left(X_{n}\right)=\sum_{i=1}^{2^{n}-1} \theta_{X_{n}}(i)=2^{n-1}\left(2^{n}-1\right)
$$

Proof. By Lemma 2.3 and analysis above, we have $\operatorname{MinL} A\left(X_{n}\right) \geq \sum_{i=1}^{2^{n}-1} \theta_{X_{n}}(i)=2^{n-1}\left(2^{n}-1\right)$. To prove the equality, we need to show that for any $X_{n} \in \ell_{n}$, for any BC structure linear arrangement $f_{n}$

$$
L A_{f_{n}}\left(X_{n}\right)=2^{n-1}\left(2^{n}-1\right) .
$$

It is direct to show that $L A_{f_{1}}\left(X_{1}\right)=1=2^{0}\left(2^{1}-1\right)$.

Suppose for $n \geq 2$, any $X_{n-1} \in \ell_{n-1}$ and any BC structure linear arrangement $f_{n-1}$,

$$
L A_{f_{n-1}}\left(X_{n-1}\right)=2^{n-2}\left(2^{n-1}-1\right) .
$$

Take any $X_{n} \in \ell_{n}$ and any $n$-dimensional BC linear arrangement $f_{n}: V\left(X_{n}\right) \rightarrow P_{n}$. Then there is $G_{1}, G_{2} \in \ell_{n-1}$ such that $X_{n}=G_{1} \oplus G_{2}$ and there are $(n-1)$-dimensional BC structure linear arrangements $f^{\prime}: V\left(G_{1}\right) \rightarrow P_{n-1}, f^{\prime \prime}: V\left(G_{2}\right) \rightarrow P_{n-1}$ such that for any $v \in V\left(G_{1}\right), f_{n}(v)=f^{\prime}(v)$, for any $v \in V\left(G_{2}\right), f_{n}(v)=f^{\prime \prime}(v)+2^{n-1}$. Then

$$
L A_{f_{n}}\left(X_{n}\right)=L A_{f^{\prime}}\left(G_{1}\right)+L A_{f^{\prime \prime}}\left(G_{2}\right)+\sum_{a \in G_{1}, b \in G_{2}}\left|P_{f_{n}}(a, b)\right| .
$$

By induction hypothesis, $L A_{f^{\prime}}\left(G_{1}\right)=L A_{f^{\prime \prime}}\left(G_{2}\right)=2^{n-2}\left(2^{n-1}-1\right)$.

By direct computation,

$$
\sum_{a \in G_{1}, b \in G_{2}}\left|P_{f_{n}}(a, b)\right|=2^{n-1}+2 \sum_{i=1}^{2^{n-1}-1} i=2^{2 n-2} .
$$

Thus for any $n$-dimensional BC structure linear arrangement $f_{n}$, we have

$$
L A_{f_{n}}\left(X_{n}\right)=2^{n-1}\left(2^{n}-1\right) .
$$

. Hence, the theorem is proved by induction. 


\section{Concluding Remarks}

In this paper, we computed the MinLA for BC graphs. Finding the other parameters, such as bandwidth, cutwidth, edge bisection and vertex bisection for BC graphs are under investigation.

\section{References}

[1] Petit, J. (2011) Addenda to the survey of layout problems, Bulletin of the EATCS, University of Patras campus, Greece, 105, 177-201.

[2] Diaz, J. Petit, J. and Serna, M. (2002) A survey of graph layout problems, ACM Computing Surveys, 34, 313-356.

[3] Raoufi, P. Rostami, H. and Bagherinezhad, H. (2013) An optimal time algorithm for minimum linear arrangement of chord graphs, Information Sciences, 238, 212-220.

[4] Harper, L.H. (1964) Optimal assignments of numbers to vertices, SIAM Journal on Applied Mathematics, 12, 131-135.

[5] Even, S. and Shiloach, Y. (1975) NP-completeness of several arrangement problems, Technical report TR-43, Department of Computer Science, Technion, Haifa.

[6] Cohen, J. Fomin, F. V. Heggernes, P. Kratsch, D. and Kucherov, G. (2006) Optimal linear arrangement of interval graphs, Lecture Notes in Computer Science LNCS, Springer, 4162, 267-279.

[7] Rajasingh, I. and Arockiaraj, M. (2011) Linear wirelength of folded hypercubes, Mathematics in Computer Science, 5(1), 101-111.

[8] Rajasingh, I. Manuel, P. Arockiaraj, M. and Rajan, B. (2013) Embeddings of circulant networks, Journal of Combinatorial Optimization, 26, 135-151.

[9] Rajasingh, I. Arockiaraj, M. Rajan, B. and Manuel, P. (2011) Circular wirelength of generalized Petersen graphs, Journal of Interconnection Networks, 12, 319-335.

[10] Arockiaraj, M. Abraham, J. Quadras, J and Shalini, A. J. (2015)Linear layout of locally twisted cubes, International Journal of Computer Mathematics, 1-11.

[11] Bezrukov, S. L. (1999) Edge isoperimetric problems on graphs, in: L. Lovsz, A. Gyarfas, G.O.H. Katona, A. Recski, L. Szekely (Eds.), Graph Theory and Combinatorial Biology, Bolyai Soc. Math. Stud., Budapest, 7, 157-197.

[12] Fan, J. and He, L. (2003) BC interconnection networks and their properties, Chinese Journal of Computers, 26(1), 84-90.

[13] Fan, J. and Lin, X. (2005) The $t / k$-diagnosability of the BC graphs, IEEE Transactions on Computers, 54, 176-184.

[14] Fan, J. Jia, X. and Lin, X. (2008) Embedding of cycles in twisted cubes with edge-pancyclic, Algorithmica, 51, 264-282. 
[15] Tan, X. Yu, S.-Z. and Park, J.-H. (2008) A note about some properties of BC graphs, Information Processing Letters, 108, 398-401.

[16] Shawn, M. L. and Paul, C. (1995) The Möbius Cubes, IEEE Transactions on Computers, 44(5), 647-659.

[17] Efe, K. (1991) A variation on the hypercube with lower diameter, IEEE Transactions on Computers, 40(11), 1312-1316.

[18] Abraham, S. and Padmanabhan, K. (1991) The twisted cube topology for multiprocessors: a study in network asymmetry, Journal of Parallel and Distributed Computing, 13(1), 104110 .

[19] Yang, X. Evans, D.J. and Megson, G.M. (2005) The locally twisted cubes, International Journal of Computer Mathematics, 82(4), 410-413.

[20] Zhou, W. Fan, J. Jia X. and Zhang, S. (2011) The spined cube: A new hypercube variant with smaller diameter, Information Processing Letters, 111(12), 561-567.

[21] Zhu, X. (2015) The Z-cubes: a hypercube variant with small diameter, arXiv:1509.06884 